\title{
Identification of cold tolerance QTLs at the bud burst stage in 211 rice landraces by GWAS
}

\author{
Caijing Li $\mathrm{i}^{1,2}$, Jindong Liu ${ }^{3}$, Jianxin Bian ${ }^{4}$, Tao Jin ${ }^{1,2}$, Baoli Zou ${ }^{1,2}$, Shilei Liu ${ }^{1,2}$, Xiangyu Zhang ${ }^{1,2}$, Peng Wang ${ }^{1,2}$, \\ Jingai Tan ${ }^{1,2}$, Guangliang Wu ${ }^{1,2}$, Qin Cheng ${ }^{1,2}$, Yanning Wang ${ }^{1,2}$, Qi Zhong ${ }^{1,2}$, Shiying Huang 1,2, \\ Mengmeng Yang ${ }^{1,2}$, Tao Huang ${ }^{1,2}$, Haohua $\mathrm{He}^{1,2^{*}}$ and Jianmin Bian ${ }^{1,2^{*}}$
}

\begin{abstract}
Background: Rice is a crop that is very sensitive to low temperature, and its morphological development and production are greatly affected by low temperature. Therefore, understanding the genetic basis of cold tolerance in rice is of great significance for mining favorable genes and cultivating excellent rice varieties. However, there have been limited studies focusing on cold tolerance at the bud burst stage; therefore, considerable attention should be given to the genetic basis of cold tolerance at this stage.

Results: In this study, a natural population consisting of 211 rice landraces collected from 15 provinces in China and other countries was used for the first time to evaluate cold tolerance at the bud burst stage. Population structure analysis showed that this population was divided into two groups and was rich in genetic diversity. Our evaluation results confirmed that japonica rice was more tolerant to cold at the bud burst stage than indica rice. A genome-wide association study (GWAS) was performed with the phenotypic data of 211 rice landraces and a 36,727 SNP dataset under a mixed linear model. Twelve QTLs $(P<0.0001)$ were identified for the seedling survival rate $(S R)$ after treatment at $4{ }^{\circ} \mathrm{C}$, in which there were five QTLS $(q S R 2-2, q S R 3-1, q S R 3-2, q S R 3-3$ and $q S R 9)$ that were colocalized with those from previous studies and seven QTLs (qSR2-1, qSR3-4, qSR3-5, qSR3-6, qSR3-7, qSR4 and qSR7) that were reported for the first time. Among these QTLS, $9 S R 9$, harboring the most significant SNP, explained the most phenotypic variation. Through bioinformatics analysis, five genes (LOC_Os09g 12440, LOC_Os09g12470, LOC_Os09g12520, LOC_Os09g12580 and LOC_Os09g12720) were identified as candidates for qSR9.

Conclusion: This natural population consisting of 211 rice landraces combined with high-density SNPs will serve as a better choice for identifying rice QTLs/genes in the future, and the detected QTLs associated with cold tolerance at the bud burst stage in rice will be conducive to further mining favorable genes and breeding rice varieties under cold stress.
\end{abstract}

Keywords: Cold tolerance, Rice landraces, GWAS, Seedling survival rate (SR), QTL

*Correspondence: hhhua64@163.com; jmbian81@126.com

${ }^{2}$ Key Laboratory of Crop Physiology, Ecology and Genetic Breeding, Nanchang 330045, Jiangxi Province, China

Full list of author information is available at the end of the article

\section{Background}

Originating in tropical and subtropical areas, rice is one of the main staple foods worldwide. Low temperature has a large impact on rice growth and cultivation. More than 15 million hectares of rice cultivated area worldwide have been affected by low temperatures. Rice is cultivated in a 
large area in China, ranging from $53^{\circ} 27^{\prime}$ to $18^{\circ} 90^{\prime}$ north latitude, especially in the provinces of the Yangtze River basin, and from 1951 to 1980, rice in the Yangtze River basin suffered from severe cold injury, with losses of 5 to 10 billion kilograms of rice every year [1]. Early rice in the Yangtze River basin in China is often affected by cold injury at the bud burst stage, resulting in a low germination rate and failure to emerge. Therefore, it is necessary to study cold tolerance in rice at the bud burst stage.

Cold tolerance is a complex quantitative trait that is often controlled by multiple genes and the environment, and researchers often use biparental populations to look for QTLs associated with cold tolerance. Researchers have found more than 250 QTLs in biparental populations using traditional QTL mapping methods during various stages of rice growth [2]. Among more than 250 QTLs, many genes throughout a variety of rice growth stages have been isolated. At the germination stage, qLTG3-1 was the first gene to be linked to germination at low temperatures [3]. During the seedling stage of rice, many QTLs/genes related to cold tolerance have been isolated, including qCTS12 [4], qCTS4 [5], qCtss11 [6], qSCT1 and $q S C T 11$ [7], qLOP2 and qPSR2-1 [8], COLD1 [9] and $q$ CTS-9 [10]. qCTS12 was the first cold tolerance gene identified at the seedling stage. COLD1 is another important gene related to cold tolerance at the seedling stage, and it is also the first cold-tolerance gene identified to be involved in signal transduction. At the booting stage of rice, several genes have been isolated, including $C t b 1$ [11], qCT8 [12], qCTB7 [13], qCTB3 [14] and qCT-3-2 [15]. $C t b 1$ is the first gene to be linked to cold tolerance at the booting stage in rice. Although biparental mapping populations have played a great role in traditional gene mapping, the construction of biparental populations entails a major investment in time and has therefore limited the number of genes identified to date [16].

To identify new genes associated with target traits, the exploration of QTLs/genes through natural populations with weak genetic relationships using GWAS has become one of the most popular methods. This method eliminates the need to construct a mapping population and can simultaneously analyze multiple alleles using recombination information from the long-term evolution of natural populations. In recent years, some studies have applied this method to study rice cold tolerance. Seventeen QTLs related to rice germination at low temperatures were detected using 63 Japanese varieties [17]. Fifty-one QTLs were mapped by GWAS with the a population of 174 rice accessions from China [18]. One hundred and thirty-two QTLs for both rice natural chilling and cold shock stresses were identified in 527 rice varieties [19]. Sixty-seven QTLs were mapped for cold stress at the seedling stage of rice, and fifty-six QTLs were newly discovered [20]. Forty-two QTLs were found to be associated with cold tolerance at the rice seedling stage, twenty of which have not been mentioned in previous reports [21]. Thirty-one QTLs related to the low-temperature germination of rice seeds at the rice germination stage were detected using 200 japonica rice varieties [22]. Forty-seven QTLs were identified for cold tolerance at the bud burst stage using 249 indica rice accessions [23]. Twenty-six QTLs were found to be related to cold tolerance at the rice seedling stage by using a core collection of landraces of rice selected from 2262 accessions of Ting's collection [24]. In addition, thirty-one distinct QTL regions were identified for low-temperature germination in a panel of 257 rice accessions from all areas of the world [25]. However, there are still few studies using GWAS to explore cold tolerance in rice at the bud burst stage. To understand the genetic mechanism of cold tolerance in rice at an early stage, it is necessary to search for QTLs related to cold tolerance in rice at the bud burst stage.

In this study, we selected 211 rice landraces from different regions to form a natural population and performed high-throughput sequencing using a microarray. The 211 rice landraces were mainly composed of indica and japonica rice, which provided abundant genetic diversity for studying the cold tolerance of rice. We treated plants from the natural population with low temperature $\left(4^{\circ} \mathrm{C}\right)$ and then recovered them at room temperature. A total of 12 QTLs, as well as 5 candidate genes for $q S R 9$, related to cold tolerance were identified by our GWAS of SR, which provided valuable genetic resources for cold tolerance research in rice and laid a solid foundation for breeding cold tolerant rice varieties.

\section{Results}

\section{Cold tolerance of the 211 rice varieties}

In our study, SR was used to evaluate cold tolerance at the bud burst stage (Table S2). Due to the abundant landrace germplasm resources, the phenotypes of rice varieties within the natural population varied greatly after $4^{\circ} \mathrm{C}$ treatment (Fig. 1). We classified cold tolerance into five levels of SR: extremely sensitive $(0 \leq X \leq 20)$, sensitive $(20<X \leq 40)$, slightly sensitive $(40<X \leq 60)$, tolerant $(60<\mathrm{X} \leq 80)$, and extremely tolerant $(80<\mathrm{X} \leq 100)$ (Table 1; Fig. 2). Of the 101 rice varieties that were extremely sensitive to low temperature, 99 were indica and 2 were japonica. Correspondingly, of the 69 rice varieties that were extremely tolerant to low temperature, 5 were indica and 64 were japonica. According to SPSS software 26.0 analysis results, the SR was significantly correlated with the indica and japonica types, and the correlation coefficient was 0.851 (Table 2), suggesting that japonica was more cold tolerant than indica at the 


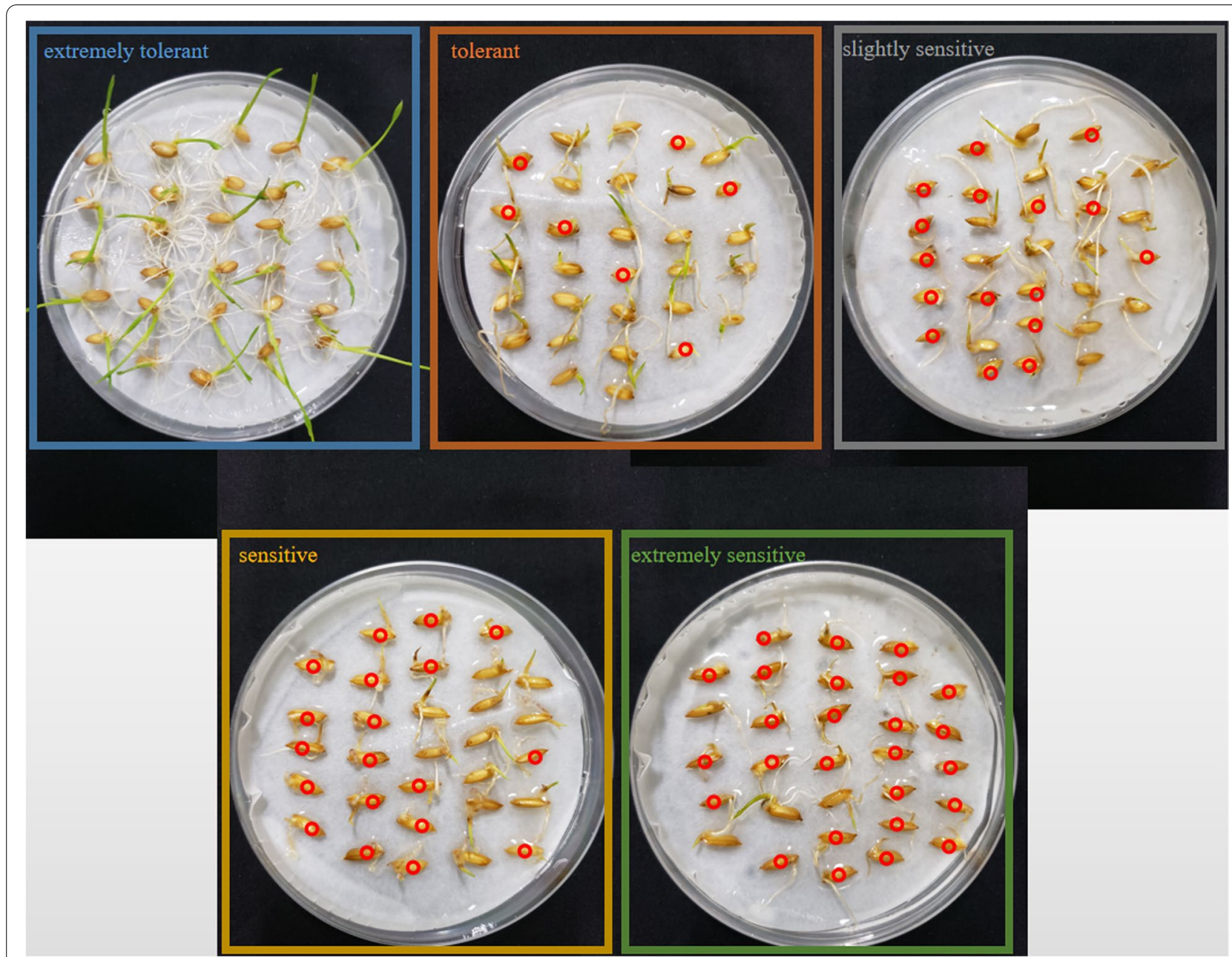

Fig. 1 Variations in cold tolerance at bud burst stage in 211 rice landraces. The five colored frame lines represent the five levels of tolerance to cold stress, and the small red circles represent the dead individuals after cold stress

Table 1 SR (seedling survival rate) after cold treatment at the bud burst stage

\begin{tabular}{llll}
\hline SR (\%) & $\begin{array}{l}\text { The total } \\
\text { number }\end{array}$ & $\begin{array}{l}\text { Number of } \\
\text { indica }\end{array}$ & $\begin{array}{l}\text { Number } \\
\text { of } \\
\text { japonica }\end{array}$ \\
\hline $0 \leq X \leq 20$ & 101 & 99 & 2 \\
$20<X \leq 40$ & 19 & 17 & 2 \\
$40<X \leq 60$ & 11 & 8 & 3 \\
$60<X \leq 80$ & 11 & 1 & 10 \\
$80<X \leq 100$ & 69 & 5 & 64 \\
\hline
\end{tabular}

bud burst stage. On the other hand, we found that the cold tolerance of rice was significantly related to its geographical distribution, and the correlation coefficient was 0.714 (Table 2). The higher the latitude was, the stronger the cold tolerance of rice, which may be due to the significant correlation between indica or japonica types and latitude (Table 2).

\section{Population structure and relative kinship}

STRUCTURE, a neighbor-joining (NJ) tree analysis, a principal component analysis (PCA) and kinship were used to analyze the population structure of the natural population based on 36,727 SNPs (Fig. 3). According to the STRUCTURE analysis, the log likelihood increased gradually from $K=1$ to $K=10$. The maximum ad hoc measure $1 \mathrm{~K}$ was observed for $\mathrm{K}=2$, which indicated that the entire population could be divided into two subgroups (Fig. 3A). The 211 rice varieties could be divided into 2 subgroups by the $\mathrm{NJ}$ tree (Fig. 3B) and the two principal components from this panel (Fig. 3C). In addition, in the relationship analysis, we found that there were two major subgroups in the 211 rice varieties 


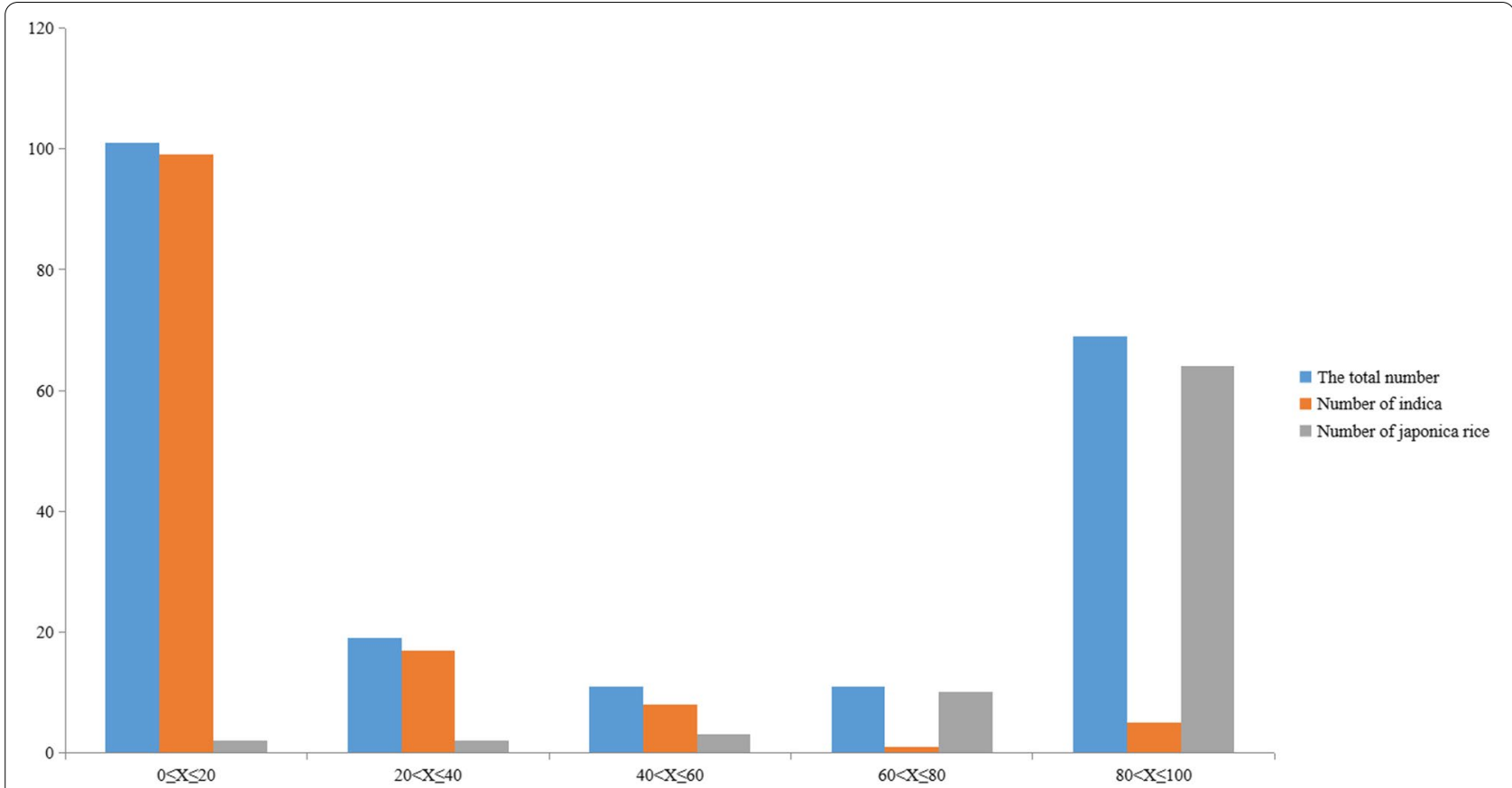

Fig. 2 Histogram of SR. The blue area represents the total number of rice samples, the orange area represents indica rice, and the gray area represents japonica rice; the horizontal axis represents the cold tolerance level

Table 2 Correlations between SR, latitude and indica or japonica type

\begin{tabular}{|c|c|c|c|}
\hline Traits & SR & Latitude & $\begin{array}{l}\text { Indica or } \\
\text { japonica } \\
\text { type }\end{array}$ \\
\hline$S R$ & & $0.714^{\mathrm{a}}$ & $0.851^{\mathrm{a}}$ \\
\hline Latitude & & & $0.910^{\mathrm{a}}$ \\
\hline $\begin{array}{l}\text { Indica or japonica } \\
\text { type }\end{array}$ & & & \\
\hline
\end{tabular}

(Fig. 3D), suggesting that landraces population germplasm resources had abundant genetic diversity, which was beneficial for performing GWAS.

\section{GWAS analysis for cold tolerance at the bud burst stage}

Based on genotype and phenotype data, an association analysis was performed under a mixed linear model using the PCA and KINSHIP results as co-variables. A total of 12 QTLs (qSR2-1, qSR2-2, qSR3-1, qSR32, qSR3-3, qSR3-4, qSR3-5, qSR3-6, qSR3-7, qSR4, $q S R 7$ and $q S R 9)$ were identified in SR with well-fitted quantile-quantile (Q-Q) plots $(p<0.0001)$ (Fig. 4 and Fig. 5; Table 3). A QTL, $q S R 2-2$, on chromosome 2 overlapped with OsWRKY71. Another QTL on chromosome 2, $q S R 2-1$, has not been previously reported.
On chromosome 3, there were three QTLs overlapping with those from previous studies, namely, qSR3-1, qSR3-2 and qSR3-3, and the other four QTLs on chromosome 3 were newly discovered and have not been reported by previous studies. The remaining two QTLs on chromosomes 4 ( $q S R 4)$ and 7 ( $q S R 7)$ were also newly discovered in this study. In addition, we also found a QTL on chromosome 9, qSR9, which harbored the most significant SNP and explained most of the phenotypic variation. This QTL is located at approximately 7.1 Mb on chromosome 9 and colocalizes with $\operatorname{clr} 9$.

\section{Candidate gene analysis}

Among these QTLs, we conducted further candidate genetic analysis of $q S R 9$. According to the LD decay analysis, a 244-kb region was identified as the candidate region (Fig. 6). There were 39 genes in this region, including 3 hypothetical proteins, 4 transposon proteins, 7 retrotransposon proteins and 16 functionally annotated genes (Table S3). To identify possible candidate genes, we analyzed the homology between these 39 genes and 20 characterized cold-tolerance genes. LOC_OsO9g12440, LOC_Os09g12470, LOC_Os09g12520, LOC_Os09g12580 and $L O C_{-} O s 09 g 12720$ were found to have a high degree of homology with COLD1, Ctb1, LTG1, OsWRKY71 and OsbZIP73 (Fig. 7; Table 4). 


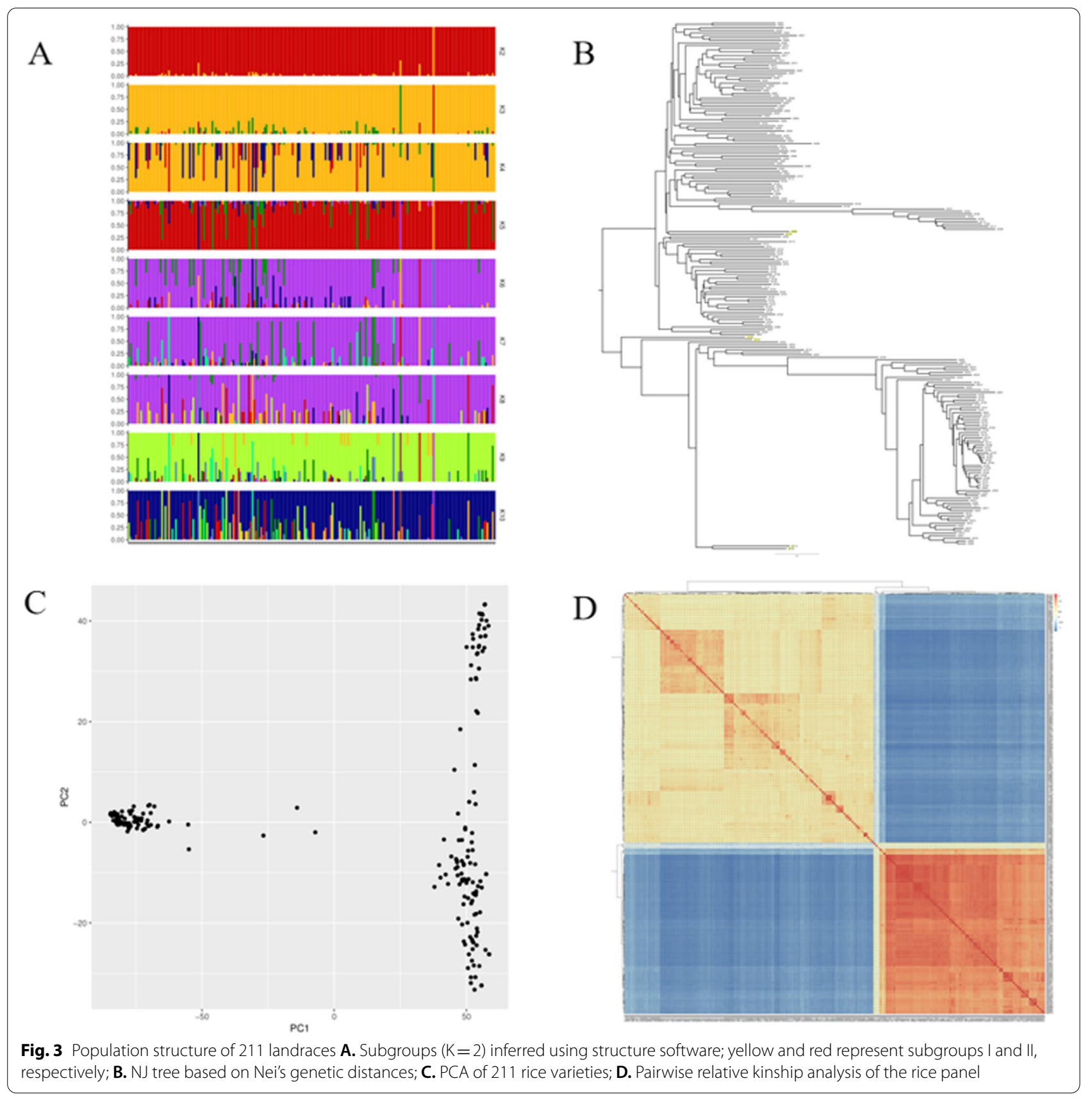

\section{Discussion}

\section{Population structure and phenotypic assessment} of a natural population

For this study, GWAS was used to reveal the complicated genetic variations related to cold tolerance. However, population structure is an important factor affecting the results of GWAS and increases the false positive rate. In this study, a natural population consisting of 211 rice landraces (130 indica rice and 81 japonica rice) was used to assess cold tolerance in rice at the bud burst stage. Most of the rice varieties come from 15 provinces in China, with three from Japan and one from the Philippines. The geographical regions span from $15^{\circ}$ to $48^{\circ}$ north latitude, including temperate zones, tropics and subtropics. This natural population is newly constructed and has rich genetic diversity. Population structure analysis divided the natural population into two groups. Subsequently, the results of PCA and NJ tree construction support the low relatedness identified by the kinship analysis (Fig. 3), which makes these data suitable for GWAS. 


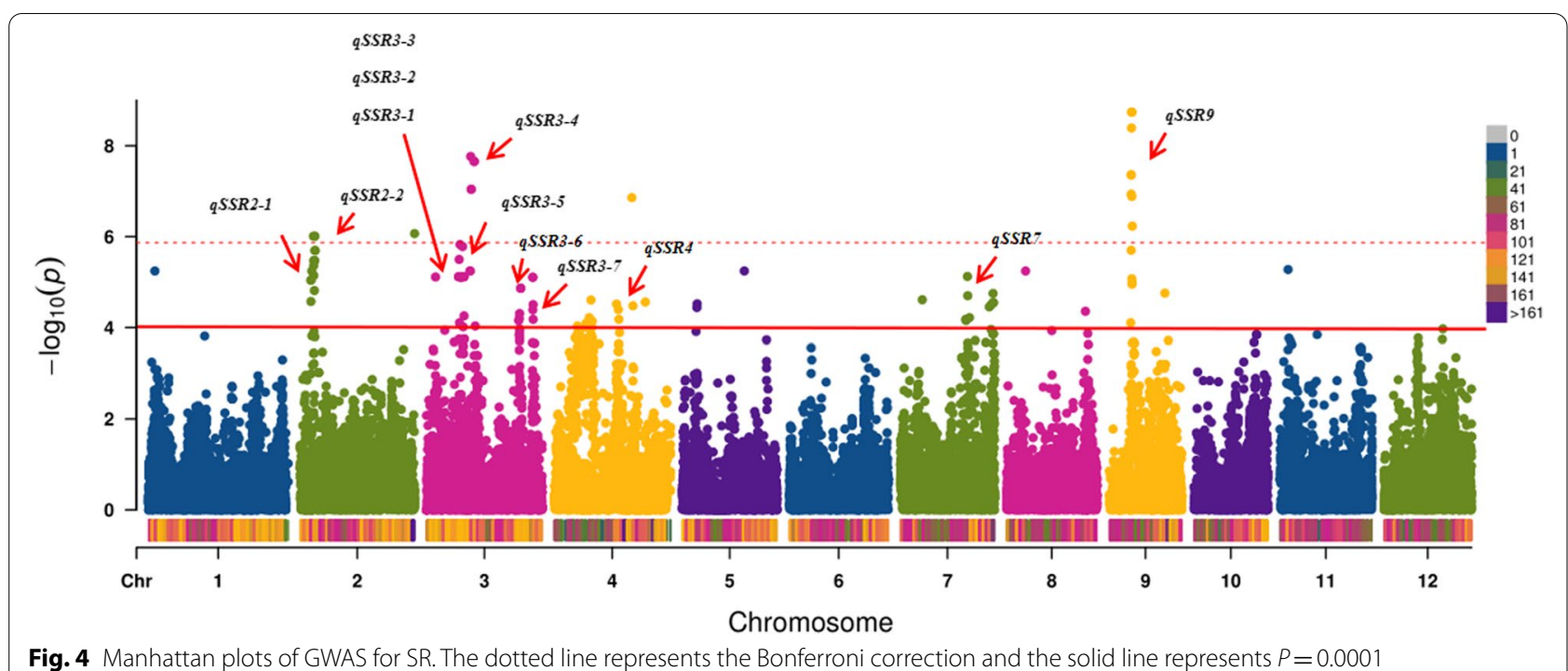

Fig. 4 Manhattan plots of GWAS for SR. The dotted line represents the Bonferroni correction and the solid line represents $P=0.0001$

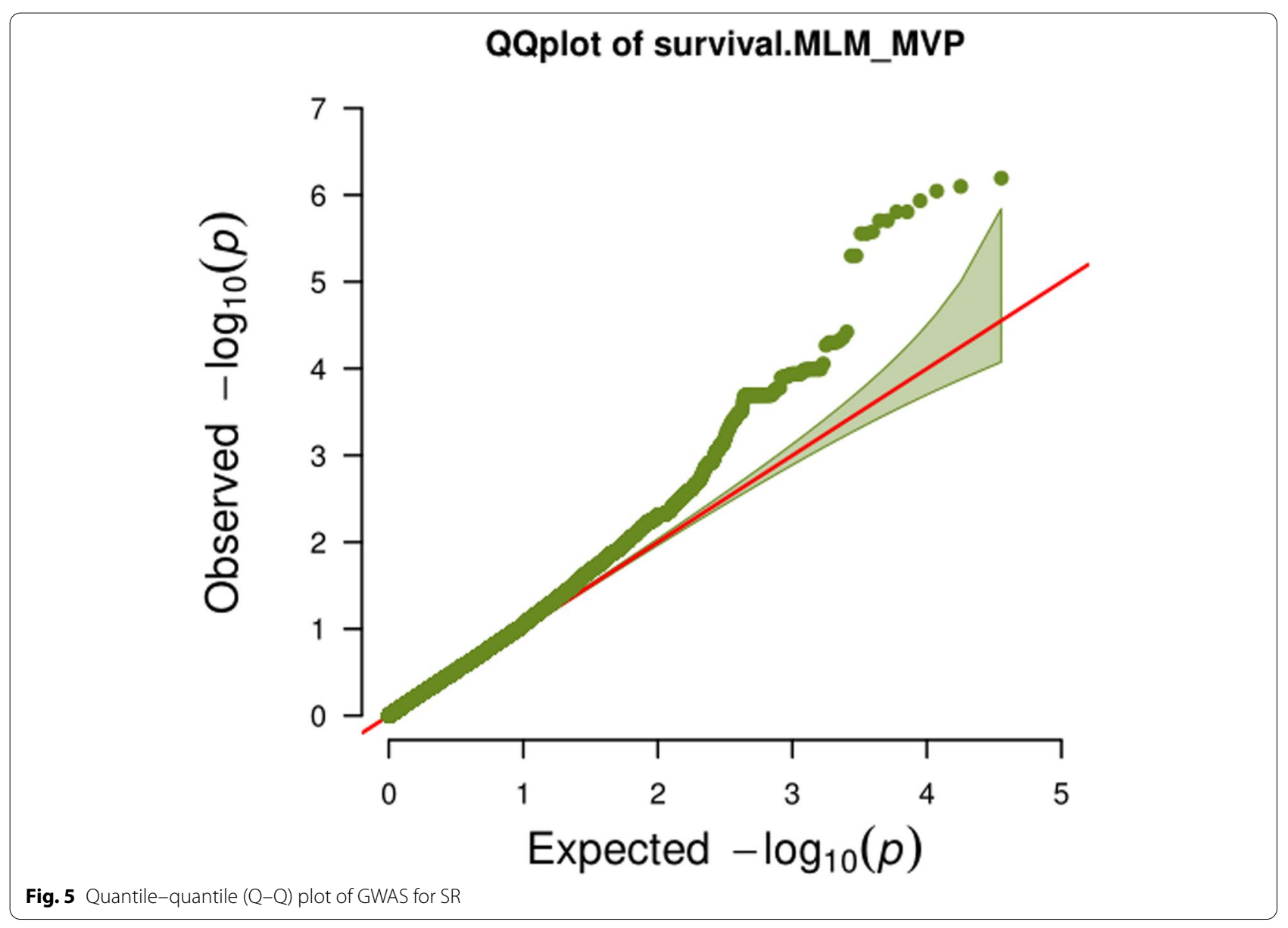


Table 3 Summary of the significant SNPs detected by GWAS and the overlapping QTLs/genes reported previously

\begin{tabular}{|c|c|c|c|c|c|c|}
\hline QTL ID & Trait & Chr. & Peak SNPs & $P$ value & $\mathrm{R}^{2}$ & Previous QTL/genes \\
\hline QSR2-1 & seedling survival rate & 2 & $3,379,509$ & 8.99E-06 & 0.076575784 & \\
\hline qSR2-2 & seedling survival rate & 2 & $4,435,145$ & $9.70 \mathrm{E}-07$ & 0.085670420 & OsWRKY71 [26] \\
\hline qSR3-1 & seedling survival rate & 3 & $10,230,171$ & $3.16 \mathrm{E}-06$ & 0.071228227 & qLVG3 [27] \\
\hline qSR3-2 & seedling survival rate & 3 & $10,552,889$ & $1.48 \mathrm{E}-06$ & 0.068345879 & qLVG3 [27] \\
\hline \multirow[t]{3}{*}{ qSR3-3 } & seedling survival rate & 3 & $11,316,517$ & 1.64E-06 & 0.057976301 & qLVG3 [27] \\
\hline & & & & & & OsMYB2 [28] \\
\hline & & & & & & OsCIPKO3 [29] \\
\hline qSR3-4 & seedling survival rate & 3 & $13,860,165$ & $1.72 \mathrm{E}-08$ & 0.111646171 & \\
\hline qSR3-5 & seedling survival rate & 3 & $15,042,189$ & $2.19 \mathrm{E}-08$ & 0.094111949 & \\
\hline qSR3-6 & seedling survival rate & 3 & $29,271,308$ & 4.89E-05 & 0.038274826 & \\
\hline qSR3-7 & seedling survival rate & 3 & $33,444,741$ & 7.77E-06 & 0.048865413 & \\
\hline qSR4 & seedling survival rate & 4 & $11,487,556$ & $2.46 \mathrm{E}-05$ & 0.03888723 & \\
\hline qSR7 & seedling survival rate & 7 & $29,111,646$ & $1.77 \mathrm{E}-05$ & 0.039475464 & \\
\hline qSR9 & seedling survival rate & 9 & $7,106,185$ & 4.07E-09 & 0.136902573 & clr9[30] \\
\hline
\end{tabular}

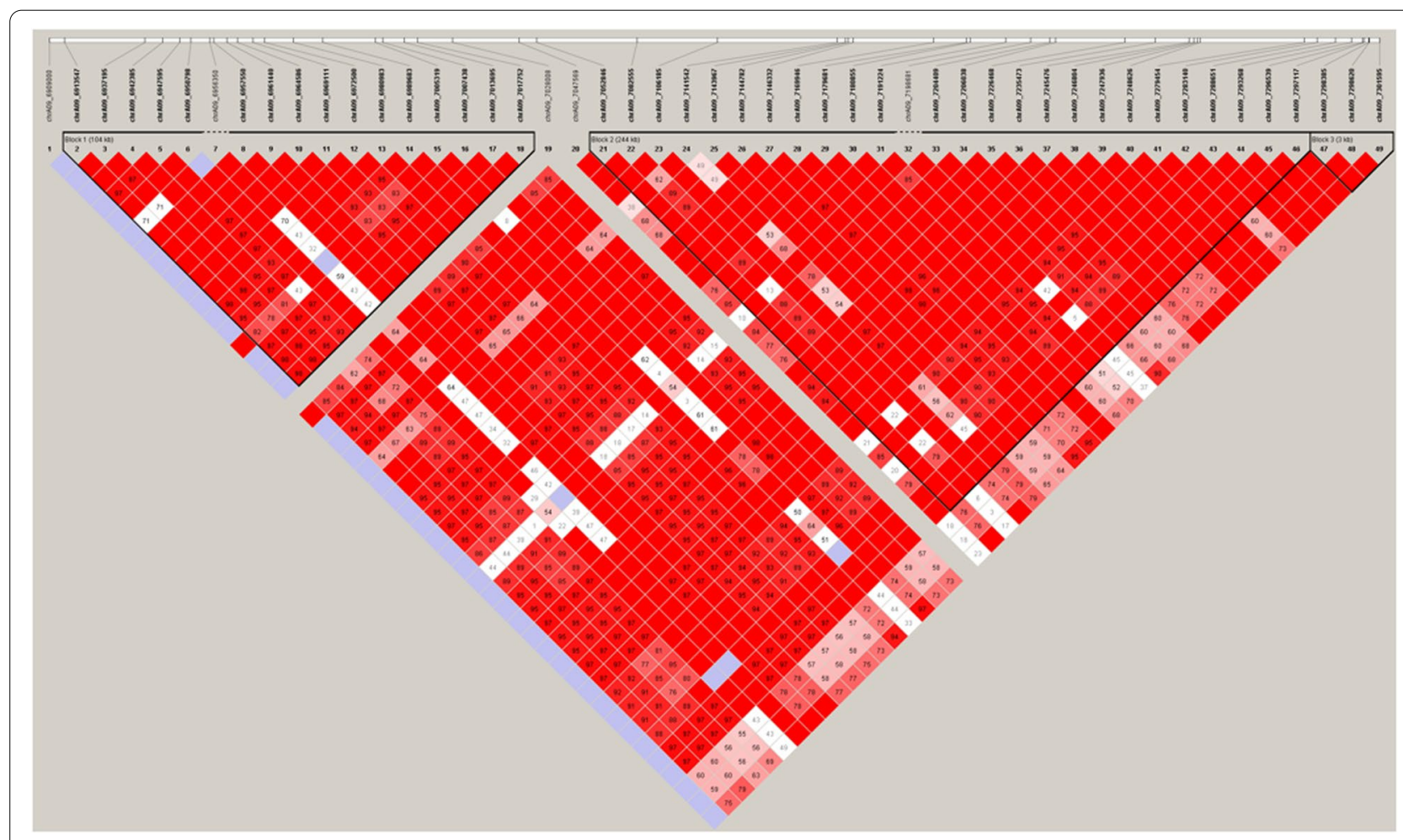

Fig. 6 LD heatmap around the peak on chromosome 9

We used the SR as the indicator to evaluate the cold tolerance of natural populations. The results show that the SR ranged from 0 to $100 \%$ (Table S2); indica rice is extremely sensitive to temperature, and its SR is low after cold treatment. Some indica rice varieties even died in the recovery time. Japonica rice is cold tolerant, and the SR of most japonica rice varieties is above $90 \%$. This result showed that japonica rice was more tolerant to cold than indica rice at the bud burst stage. 


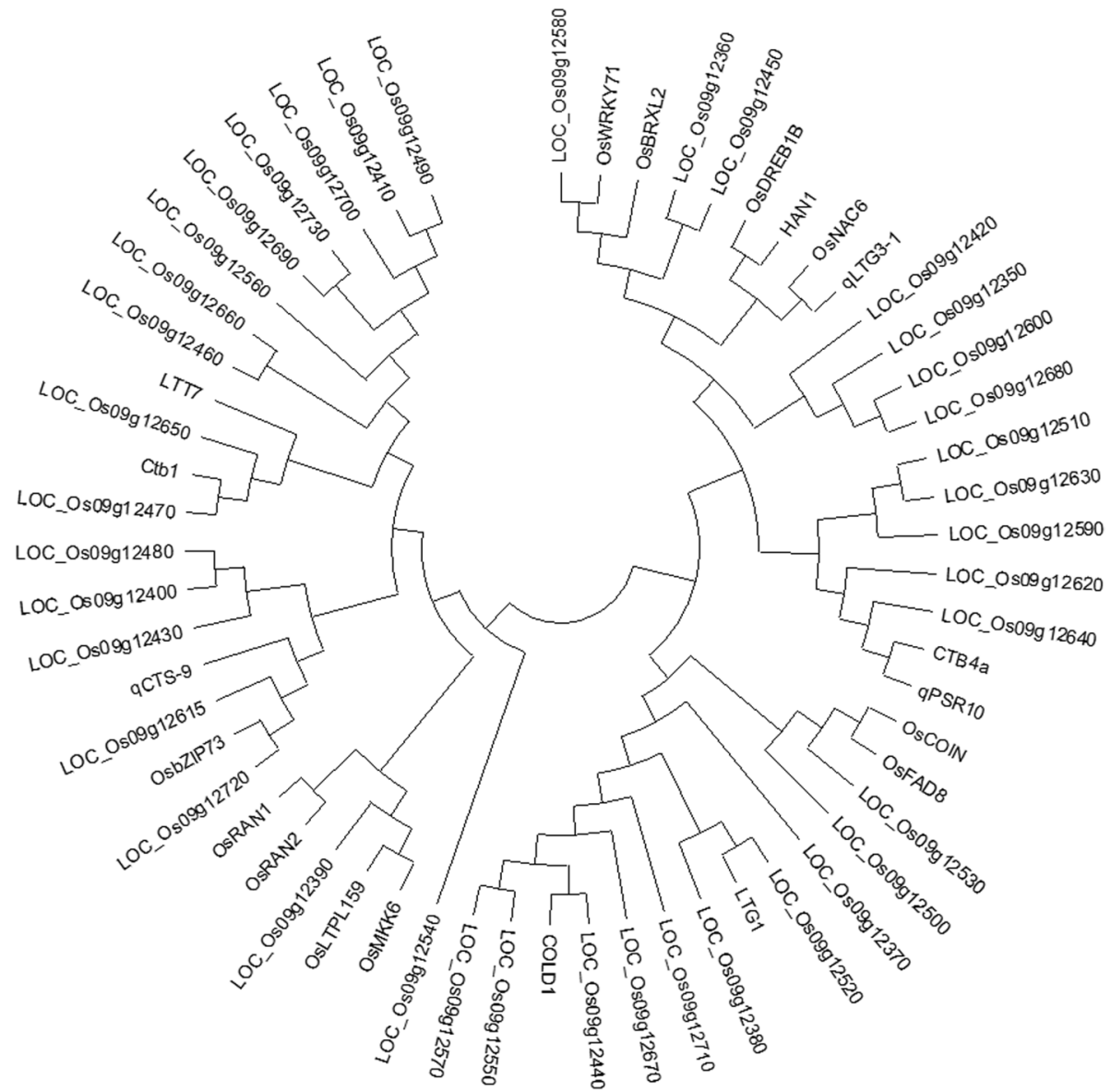

Fig. 7 Homology analysis among of 59 genes (39 putative genes and 20 reference genes)

Table 4 Candidate genes in the qSR9 region

\begin{tabular}{|c|c|c|c|}
\hline QTL & Putative genes & $\begin{array}{l}\text { Putative protein } \\
\text { functions }\end{array}$ & Reference genes \\
\hline \multirow[t]{5}{*}{ qSR9 } & LOC_Os09g12440 & $\begin{array}{l}\text { retrotransposon protein, } \\
\text { putative, unclassified, } \\
\text { expressed }\end{array}$ & COLD1 \\
\hline & LOC_Os09g12470 & hypothetical protein & CTB1 \\
\hline & LOC_Os09g12520 & hypothetical protein & LTG1 [31] \\
\hline & LOC_Os09g12580 & expressed protein & OsWRKY71 \\
\hline & LOC_Os09g12720 & $\begin{array}{l}\text { zinc finger, } \mathrm{C} 3 \mathrm{HC} 4 \text { type } \\
\text { domain containing } \\
\text { protein, expressed }\end{array}$ & OsbZIP73 [32] \\
\hline
\end{tabular}

\section{Identification of QTLs/candidate genes controlling cold tolerance at the bud burst stage}

In this study, we found 12 QTLs for SR after cold treatment. Among these QTLs, seven (qSR2-1, qSR3-4, $q S R 3-5, q S R 3-6, q S R 3-7, q S R 4$ and $q S R 7)$ were reported for the first time, and the other five QTLs ( $q S R 2-2$, $q S R 3-1, q S R 3-2, q S R 3-3$ and $q S R 9)$ were colocalized with QTLs from previous studies. The peak SNP for qSR2-2 was located at $4.4 \mathrm{Mb}$ on chromosome 2 . This region overlapped with $O s W R K Y 71$, which is a transcriptional suppressor that encodes GA signaling in aleurone cells and cold tolerance. qSR3-1, qSR3-2 and qSR3-3 were colocalized with $q L V G 3$, a QTL for low-temperature vigor during germination. In the $q L V G 3$ region, there were two characterized cold tolerance genes (OsMYB2 and OsCIPK03). OsMYB2 is a MYB transcription factor and plays a regulatory role in the tolerance of rice to salt, cold injury and dehydration stress, while OsCIPKO3 is a calcineurin B-like protein-interacting protein kinase. The overexpression of OsCIPKO3 in transgenic plants significantly improved their tolerance to cold stress. The QTL qSR9 explained the largest phenotypic variation in our study, overlapping with $\operatorname{clr} 9$, which is a QTL associated with the culm length growth rate under cold stress; however, knowledge about the candidate genes underlying qSR9 is still lacking.

The abundant SNP dataset for our natural population obtained through a chip strategy makes it feasible 
to locate $q S R 9$ to a small genomic region. The candidate gene analysis showed that there were 39 candidate genes underlying $q S R 9$. Among these candidate genes, five genes (LOC_Os09g12440, LOC_Os09g12470, LOC_ Os09g12520, LOC_Os09g12580 and LOC_Os09g12720) might be the target genes because these five candidate genes are homologous to the characterized cold tolerance genes COLD1, Ctb1, LTG1, OsWRKY71 and OsbZIP73, respectively (Fig. 7). For these characterized cold tolerance genes, COLD1 encodes a G protein signal regulator and can interact with RGA1, the alpha subunit of the $G$ protein, to sense low temperature, activate $\mathrm{Ca}^{2+}$ channels, and enhance the activity of the $\mathrm{G}$ protein GTP enzyme to enhance the cold tolerance of rice. $C t b 1$ encodes an F-box protein, which interacts with the E3 ubiquitin ligase subunit SKP1 and is involved in cold tolerance at the booting stage. LTG1 encodes a casein kinase, regulates the cold response in rice, affects auxin transport, synthesis and signal transduction, and positively regulates the low-temperature tolerance of rice during the vegetative growth period. OsbZIP73 ${ }^{\text {Jap }}$ is upregulated by low temperature and the plant hormone abscisic acid (ABA), suggesting that OsbZIP73 is involved in ABA-dependent lowtemperature signaling pathways. However, other genes underlying $q S R 9$ cannot be ruled out, such as $L O C_{-}$ Os09g12360, LOC_Os09g12390, LOC_Os09g12450, LOC_Os09g12615, LOC_Os09g12640 and LOC_ Os09g12650. Although these genes are not homologous to the these characterized cold tolerance genes, they are very homologous to other characterized cold tolerance genes (Fig. 7). Further studies, such as gene complementation analysis, are necessary to elucidate which allele is more favorable.

\section{Conclusions}

In this study, a natural population consisting of 211 rice landraces was used to assess cold tolerance at the bud burst stage by using GWAS under a mixed linear model. Twelve QTLs were detected on chromosomes 2, 3, 4, 7, and 9, and five genes (LOC_OsO9g12440, LOC_ Os09g12470, LOC_Os09g12520, LOC_Os09g12580 and $L O C \_O s 09 g 12720$ ) might be the target genes for $q S R 9$ after candidate gene analysis. These QTLs/genes will be conducive to further mining favorable genetic resources and breeding rice varieties. The highlight of this study is the combination of previously unstudied rice landraces with the latest sequencing technology and the discovery of a number of new QTLs related to cold tolerance in rice that will assist in the breeding of new rice varieties with cold tolerance.

\section{Methods}

\section{Plant material}

A natural population of 211 rice varieties selected from International Rice Research Institute (https://www.irri. $\mathrm{org} /$ ) was used to evaluate the cold tolerance of rice at the bud burst stage (Table S1). These varieties were mainly collected from 15 different provinces in China as well as from the Philippines and Japan. Their geographical range spans from $15^{\circ}$ to $48^{\circ}$ north latitude, including temperate, tropical and subtropical regions. Of the 211 rice varieties, 81 were classified as japonica rice, and 130 were classified as indica rice. The rice materials were collected in accordance with local laws without any conflict of interest. The population was developed in the experimental field at Jiangxi Agricultural University in Nanchang, Jiangxi Province, and Linwang, Hainan Province, for more than two generations.

\section{Cold tolerance evaluation at the bud burst stage}

The tested seeds were placed in an oven at $45^{\circ} \mathrm{C}$ for $48 \mathrm{~h}$ to break seed dormancy. The seeds were disinfected with sodium hypochlorite solution and rinsed with sterile water three times. Next, the seeds were soaked in water for approximately $48 \mathrm{~h}$ and allowed to germinate for $24 \mathrm{~h}$. Thirty seeds with $5 \mathrm{~mm}$ coleoptile lengths were selected and placed in a petri dish with two sheets of filter paper. The petri dishes were placed in a growth incubator at $4{ }^{\circ} \mathrm{C}$ and treated in darkness for 10 days. After cold treatment, the petri dishes containing the seeds were placed in an incubator at $25^{\circ} \mathrm{C}$ (14-h light/10-h dark) and recovered for 5 days. The seedling survival rate (SR) was calculated after 5 days of recovery of growth and used as the indicator of cold tolerance at the bud burst stage. Seedling survival rate $(\%)=$ number of surviving seeds $/ 30 \times 100$. All experiments were repeated three times.

\section{GWAS mapping of rice cold tolerance QTLs/genes at the bud burst stage}

Tassel 5.0 software was used for the GWAS of rice cold tolerance at the bud burst stage. Principal component analysis (PCA) and kinship analysis were performed using a 36,727 SNP genotype dataset. After filtering, standardized phenotype data, SNP data and principal component data were merged, and GWAS was performed with kinship data as covariables under a mixed linear model. Plotting was performed with the R package R_MVP. QTL regions were identified when 3 or more significant SNPs occurred within a $400 \mathrm{~kb}$ interval. Candidate gene analyses of $q S R 9$ were conducted with RAP-DB (https://rapdb.dna.affrc.go.jp/) and NCBI (https://www. ncbi.nlm.nih.gov/). The DNA sequences of the putative 
genes and cold tolerance genes were downloaded from NCBI and analyzed with MEGA-X software (https:// www.megasoftware.net/).

\section{Statistical and genetic analyses}

A correlation analysis of the SR, latitude and indica or japonica type was carried out with SPSS Statistics 26 software.

\section{Genomic DNA extraction, sequencing and genotyping}

The CTAB method was used to extract DNA from approximately $100 \mathrm{mg}$ of fresh young leaves, and the quantity and quality of DNA were measured using a Denovix DS-11 spectrophotometer. In addition, purity was determined by electrophoresis of the DNA for $1 \mathrm{~h}$ at $60 \mathrm{~V}$ in a $1 \%$ agarose gel. The $50 \mathrm{~K}$ rice gene SNP microarray 'OsSNPNKs' was used for genotyping. The SNPs on the microarrays were evenly distributed throughout the genome, with an average distance of $<1 \mathrm{~kb}$ between SNPs. Genotyping was based on Affymetrix $\mathrm{AXIOM}^{\circledR} 2.0$. The Target Prep Protocol QRC (P/N 702990) Kit was used for DNA amplification, DNA fragmentation, microarray hybridization, DNA binding single base extension, and signal amplification. Staining and scanning were performed using a GeneTitan ${ }^{\circledR}$ multichannel instrument [33].

\section{Abbreviations}

SR: Seedling survival rate; QTL: Quantitative trait loci; GWAS: Genome-wide association study; SNP: Single nucleotide polymorphism; PCA: Principal component analysis; NJ: Neighbor-joining.

\section{Supplementary Information}

The online version contains supplementary material available at https://doi. org/10.1186/s12870-021-03317-7.

\author{
Additional file 1: Table S1. Geographical distribution and indica or \\ japonica type of 211 rice varieties \\ Additional file 2: Table S2. SR of the 211 rice varieties \\ Additional file 3: Table S3. 39 candidate genes for $q S R 9$ \\ Additional file 4: Table S4. The SNPs data used for performed GWAS
}

\section{Acknowledgments}

We thank the anonymous referees for their critical comments on this manuscript.

\section{Authors' contributions}

$C, L$ is the first author of this article, designed and performed experiments, analyzed data and wrote the manuscript. J. L, J. B participated in analysis data. T. J, B. Z, J. T, X. Z, P.W, G. W, Q. C, Y. W, Q. Z, S. H, M. Y, T. H, S. L, C.L participated in performing experiments. H.H and J.B. conceived and supervised the experiments. All authors reviewed the manuscript. The author(s) read and approved the final manuscript.

\section{Funding}

This research was supported by grants (2016YFD0101801) from The National Key Research and Development Program of China and Technology
Department and grants (20192BCB23010, 20192ACBL20017) from Project of Science and Technology Department of Jiangxi Province and grant (202010410100, 202010410106) from National Undergraduate Training Program for Innovation and Entrepreneurship. These funding institutions provided financial support for material collection, high-throughput sequencing and data analysis in this study.

\section{Availability of data and materials}

The sequencing data generated in this study were deposited in the databases (https://snp-seek.irri.org/). In addition, we have also sorted out a genotype data Table for GWAS based on the sequencing data, please refer to Table S4 for details.

\section{Declarations}

Ethics approval and consent to participate

Not applicable.

\section{Consent for publication}

Not applicable.

\section{Competing interests}

The authors declare that the research was conducted in the absence of any commercial or financial relationships that could be construed as a potential conflict of interest.

\section{Author details}

${ }^{1}$ Key Laboratory of Crop Physiology, Ecology and Genetic Breeding, Ministry of Education, Nanchang 330045, Jiangxi Province, China. ${ }^{2}$ Key Laboratory of Crop Physiology, Ecology and Genetic Breeding, Nanchang 330045, Jiangxi Province, China. ${ }^{3}$ Agricultural Genomics Institute in Shenzhen, Chinese Academy of Agricultural Sciences, Shenzhen 518000, Guangdong Province, China. ${ }^{4}$ Peking University Institute of Advanced Agricultural Sciences, Weifang 261325, Shandong Province, China.

Received: 9 March 2021 Accepted: 4 November 2021

Published online: 20 November 2021

\section{References}

1. Dai LY, Ye CR, Yu TQ, Xu FR. Studies on cold tolerance of rice, Oryza sativa L:I. Description on types of cold injury and classifications of evaluation methods on cold tolerance in rice. Southwest China J Agric Sci. 2002;15(1):41-5.

2. Zhang Q, Chen Q, Wang S. Rice and cold stress: methods for its evaluation and summary of cold tolerance-related quantitative trait loci. Rice. 2014;7:24.

3. Fujino K, Sekiguchi H, Matsuda Y, Sugimoto K, Ono K, Yano M. Molecular identification of a major quantitative trait locus, aLTG3-1, controlling low-temperature germinability in rice. Proc Natl Acad Sci U S A. 2008;105(34):12623-8.

4. Andaya VC, Tai TH. Fine mapping of the aCTS12 locus, a major QTL for seedling cold tolerance in rice. Theor Appl Genet. 2006;113:467-75.

5. Andaya VC, Tai TH. Fine mapping of the qCTS4 locus associated with seedling cold tolerance in rice (Oryza sativa L.). Mol Breed. 2007;20:349-58.

6. Koseki M, Kitazawa N, Yonebayashi S, Maehara Y, Wang ZX, Minobe Y. Identification and fine mapping of a major quantitative trait locus originating from wild rice, controlling cold tolerance at the seedling stage. Mol Gen Genomics. 2010;284:45-54.

7. Kim SM, Suh JP, Lee CK, Lee JH, Kim YG, Jena KK. QTL mapping and development of candidate gene-derived DNA markers associated with seedling cold tolerance in rice (Oryza sativa L.). Mol Gen Genomics. 2014:289:333-43.

8. Xiao N, Huang WN, Li AH, Gao Y, Li YH, Pan CH, et al. Fine mapping of the qLOP2 and aPSR2-1 loci associated with chilling stress tolerance of wild rice seedlings. Theor Appl Genet. 2015;128:173-85.

9. Ma Y, Dai $X$, Yunyuan $X$, Luo W. COLD1 confers chilling tolerance in rice. Cell. 2015;160:1209-21. 
10. Zhao J, Zhang S, Dong J. A novel functional gene associated with cold tolerance at the seedling stage in rice. Plant Biotechnol J. 2017;15(9):1141-8.

11. Saito K, Hayano-Saito Y, Maruyama-Funatsuki W, Sato Y, Kato A. Physical mapping and putative candidate gene identification of a quantitative trait locus $C t b 1$ for cold tolerance at the booting stage of rice. Theor Appl Genet. 2004;109:515-22.

12. Kuroki M, Saito K, Matsuba S, Yokogami N, Shimizu H, Ando I, et al. A quantitative trait locus for cold tolerance at the booting stage on rice chromosome 8. Theor Appl Genet. 2007;115:593-600.

13. Zhou L, Zeng $Y$, Zheng $W$, Tang $B$, Yang $S$, Zhang $H$, et al. Fine mapping a QTL qCTB7 for cold tolerance at the booting stage on rice chromosome 7 using a near-isogenic line. Theor Appl Genet. 2010;121:895-905.

14. Shirasawa S, Endo T, Nakagomi K, Yamaguchi M, Nishio T. Delimitation of a QTL region controlling cold tolerance at booting stage of a cultivar, 'Lijiangxintuanheigu', in rice, Oryza sativa L. Theor Appl Genet. 2012;124:937-46.

15. Zhu Y, Chen K, Mi X, Chen T, Ali J, Ye G, et al. Identification and fine mapping of a stably expressed QTL for cold tolerance at the booting stage using an interconnected breeding population in rice. PLoS One. 2015;10:e0145704.

16. Bian JM, Jiang L, Liu LL, Wei XJ. Construction of a new set of rice chromosome segment substitution lines and identification of grain weight and related traits QTLs. Breed Sci. 2010;60(4):305-13.

17. Fujino K, Obara M, Shimizu T, Koyanagi KO, Ikegaya T. Genome-wide association mapping focusing on a rice population derived from rice breeding programs in a region. Breed Sci. 2015;65:403-10.

18. Pan $Y$, Hong ZH. Genetic analysis of cold tolerance at the germination and booting stages in rice by association mapping. PLoS One. 2015;10:e0120590.

19. LvY, Guo ZL, Li XK, Ye HY, Li XH, Xiong LZ. New insights into the genetic basis of natural chilling and cold shock tolerance in rice by genome-wide association analysis. Plant Cell Environ. 2016;39:556-70.

20. Wang D, Liu J, Li C. Genome-wide association mapping of cold tolerance genes at the seedling stage in rice. Rice. 2016;9:61.

21. Shakiba E, Edwards JD, Jodari F. Genetic architecture of cold tolerance in rice (Oryza sativa) determined through high resolution genome-wide analysis. PLoS One. 2017;12:e0172133.

22. Sales E, Viruel J, Domingo C, Marqués L. Genome wide association analysis of cold tolerance at germination in temperate japonica rice (Oryza sativa L.) varieties. PLoS One. 2017;12(8):e0183416.
23. Zhang M, Ye J, Xu Q. Genome-wide association study of cold tolerance of Chinese indica rice varieties at the bud burst stage. Plant Cell Rep. 2018:37:529-39.

24. Song J, Li J, Sun J, Hu T, Wu A, Liu S, et al. Genome-wide association mapping for cold tolerance in a Core collection of Rice (Oryza sativa L.) landraces by using high-density single nucleotide polymorphism markers from specific-locus amplified fragment sequencing. Front Plant Sci. 2018;9:875.

25. Thapa R, Tabien R, Thomson M, Septiningsih E. Genome-wide association mapping to identify genetic loci for cold tolerance and cold recovery during germination in Rice. Front Genet. 2020:11:22.

26. Kim CY, Vo K, T X, Nguyen C.D. Functional analysis of a cold-responsive rice WRKY gene, OsWRKY71. Plant Biotechnol Rep. 2016;10:13-23.

27. Han LZ, Zhang YY, Qiao YL, Cao GL, Zhang SY, Kim JH, et al. Genetic and QTL analysis for low-temperature vigor of germination in rice. Acta Genet Sin. 2006;33:998-1006.

28. Yang A, Dai X, Zhang W. A R2R3-type MYB gene, OsMYB2, is involved in salt, cold, and dehydration tolerance in rice. J Exp Bot. 2012;63(7):2541-56

29. Yong $X$, Yue H, Li X. Characterization of stress-responsive CIPK genes in Rice for stress tolerance improvement. Plant Physiol. 2007;144(3):1416-28.

30. Oh CS, Choi YH, Lee SJ, Yoon DB, Moon HP, Ahn SN. Mapping of quantitative trait loci for cold tolerance in weedy Rice. Breeding Sci. 2004:54:373-80.

31. Lu G, Wu FQ, Wu W. Rice $L T G 1$ is involved in adaptive growth and fitness under low ambient temperature. Plant J. 2014;78(3):468-80.

32. Liu C, Ou S, Mao B, Wei W. Early selection of bZIP73 facilitated adaptation of japonica rice to cold climates. Nat Commun. 2018;9(1):3302-12.

33. Rohilla M, Singh N, Mazumder A. Genome-wide association studies using $50 \mathrm{~K}$ rice genic SNP chip unveil genetic architecture for anaerobic germination of deep-water rice population of Assam, India. Mol Genet Genomics. 2020;295:1211-26.

\section{Publisher's Note}

Springer Nature remains neutral with regard to jurisdictional claims in published maps and institutional affiliations.
Ready to submit your research? Choose BMC and benefit from:

- fast, convenient online submission

- thorough peer review by experienced researchers in your field

- rapid publication on acceptance

- support for research data, including large and complex data types

- gold Open Access which fosters wider collaboration and increased citations

- maximum visibility for your research: over 100M website views per year

At BMC, research is always in progress.

Learn more biomedcentral.com/submissions 